\title{
Evaluation of appropriateness of second-generation 320-row computed tomography for coronary artery disease
}

\author{
Daisuke Utsunomiya ${ }^{1 *}$, Seitaro Oda ${ }^{1}$, Hideaki Yuki ${ }^{1}$, Megumi Yamamuro ${ }^{2}$, Kenichi Tsujita ${ }^{2}$, Yoshinori Funama ${ }^{3}$, \\ Morikatsu Yoshida', Masafumi Kidoh', Hisao Ogawa ${ }^{2}$ and Yasuyuki Yamashita ${ }^{1}$
}

\begin{abstract}
The influence of newer-generation $C T$ on the clinical indications and appropriateness of cardiac $C T$ has not been adequately surveyed. We aimed to evaluate the distribution of appropriateness ratings and test the outcomes of cardiac CT using second-generation 320-row CT. The 2010 appropriate use criteria (AUC) were applied at the point of service to a consecutive series of patients $(N=309)$ who were referred for cardiac $C T$. The $C T$ indication was determined based on interviews and medical records. The proportions of patients within the categories of appropriate (A), uncertain (U), inappropriate (I), and not covered were described. The prevalence of significant coronary artery disease (CAD) was also compared among the categories. The proportions were 49.2\%, 25.9\%, and 20.7\% for appropriate, uncertain, and inappropriate indication, respectively. The indication that was not covered was only $4.2 \%$. Significant CAD was more frequently observed for uncertain- than appropriate indication ( $42.5 \%$ vs $27.6 \% ; P=0.03$ ), although the number of significant stenosed segments was not different $(P=0.13)$. The recent advancement of cardiac $C T$ increased the proportion of uncertain scans, which were associated with a high prevalence of significant CAD.
\end{abstract}

Keywords: Cardiac; CT-Angiography; Heart; Appropriate use criteria

\section{Introduction}

The recent development of 64-row or more CT provides high-image quality and diagnostic accuracy for cardiac CT, leading to increasing applications for patients with suspected coronary artery disease (CAD). The appropriate use criteria (AUC), developed by the American College of Cardiology and others, provide guidance regarding the appropriateness of cardiac CT for any given clinical situation (Hendel et al. 2006; Taylor et al. 2010). These criteria were developed through a formal process in which expert opinion, guided by the available evidence. The different scenarios are designed based on practical experience. They are tested against clinical trials, reviewed by experts, and rated for their appropriateness into 3 categories of appropriate (A), uncertain (U), and inappropriate (I) (Rich et al. 2012). The first AUC for cardiac CT were published in 2006, followed by an updated version in 2010 , including a more

\footnotetext{
* Correspondence: utsunomi@kumamoto-u.ac.jp

'Diagnostic Radiology, Faculty of Life Sciences, Kumamoto University, 1-1-1,

Honjo, 860-8556 Kumamoto-shi, Kumamoto, Chuo-ku, Japan

Full list of author information is available at the end of the article
}

complete classification of the conditions and a large shift in appropriate rating (Rich et al. 2012; Taylor et al. 2010; Cullen et al. 2013). Among the 93 cardiac indications in the 2010 AUC, 35 were classified as appropriate, and 58 were judged either inappropriate or uncertain (Taylor et al. 2010).

Cardiac CT has undergone further technical advancement with improved image quality and safety (Leipsic et al. 2012; Nakaura et al. 2013; Oda et al. 2014; Rich et al. 2012). The combination of prospective ECG-gated axial scan and iterative reconstruction technique drastically reduces the radiation- and contrast material dose (Rich et al. 2012; Tatsugami et al. 2012). In 2013, a second-generation 320-row CT scanner was introduced in clinical practice (Chen et al. 2013). Reportedly, the faster rotation time $(0.275 \mathrm{~s})$, wider volume coverage, iterative reconstruction, automated exposure control, and larger X-ray power generator provide excellent image quality over a wide range of body sizes and heart rates at low radiation dose (Chen et al. 2013; Khan et al. 2011). However, the 2010 AUC for cardiac CT were basically 
established on the use of 64-row CT. The impact of the newer-generation CT with more than 64-row detectors on the AUC rating of clinical indications has not been adequately investigated. We hypothesized that the lessinvasive scanning and improved image quality by the newer CT could change and widen the cardiac CT indications and appropriateness.

Thus, the purpose of our survey was to assess the distribution of appropriateness ratings and test outcomes in patients referred for less-invasive cardiac CT using the second-generation 320-row CT.

\section{Methods}

\section{Ethics statement}

The study conforms to the principles of the Declaration of Helsinki and approved by the Ethics Committee of the Faculty of Life Sciences, Kumamoto University, and written informed consent was obtained from all patients. This study was registered at the Protocol Registration System of ClinicalTrials.gov (NCT02033837).

\section{Study population}

Between January-01- and December-31-2013, we prospectively applied the 2010 AUC at the point of service to 345 consecutive patients who were referred to our hospital for cardiac CT. Patients were excluded from analysis if there was insufficient clinical information available $(\mathrm{N}=$ 6), or if they underwent cardiac CT for the evaluation of aortic disease, pulmonary embolism, or pulmonary vein anatomy because these conditions are not directly related to CAD $(\mathrm{N}=30)$. Therefore, a total of 309 patients were enrolled in this analysis.

\section{Data acquisition}

All patients underwent cardiac CT on a 320-detector CT scanner (Aquilion ONE ViSION Edition, Toshiba Medical Systems, Otawara, Japan). The acquisition parameters were: 240,280 , or $320 \times 0.5-\mathrm{mm}$ detector collimation, 275-ms tube rotation time, $270-800 \mathrm{mAs}$ tube currenttime product with automatic exposure control (noise index, 20), and 80-, 100-, or $120-\mathrm{kVp}$ tube voltage, according to the patient body mass index (BMI) $(80 \mathrm{kVp}$ for $\mathrm{BMI}<21 ; 100 \mathrm{kVp}$ for BMI 21-25; $120 \mathrm{kVp}$ for BMI $>25$ ). The CT images were reconstructed using the adaptive iterative reconstruction technique (AIDR 3D, Toshiba Medical Systems, Otawara, Japan).

\section{Cardiac CT appropriateness}

The patients were interviewed, and the medical records were reviewed to customize the cardiac CT indication. Pretest CAD probability and coronary heart disease risk were estimated using the published AUC methods. Two radiologists (10 and 6 years of experience in cardiac CT) independently classified the AUC rating of each patient as appropriate, uncertain, inappropriate or not classifiable. The presence and number of coronary segments with significant CAD were also evaluated. Two experienced radiologists consensually interpreted the coronary $\mathrm{CT}$ angiogram including axial source-, curved multiplanar reformation images, and angiographic view. Significant CAD was defined as $>50 \%$ stenosis. We also recorded the machinegenerated volume CT dose index (CTDIvol) (mGy) per examination for imaging during cardiac CT. The CTDIvol averages the radiation dose over the center slice of a CT study comprising multiple parallel slices. The data acquisition range and the dose-length product were calculated on the basis of CTDIvol and the data acquisition range. Finally, the effective radiation dose of the chest was calculated with the equation: effective dose $=$ dose-length product $\times$ 0.014 (Hausleiter et al. 2009).

\section{Statistical analyses}

Numerical data were expressed as the mean \pm standard deviation. The proportion of scans within the appropriate, uncertain, or inappropriate categories were compared with the 2010 AUC ratings using the Chi-square test. The number of vessel segments with $>50 \%$ stenosis in the three categories was compared using the TukeyKramer test. All analyses were performed using the statistical software JMP 9.0.2 (SAS Institute, Cary, NC). Pvalues of $<0.05$ were considered statistically significant.

\section{Results}

\section{Demographic variables}

The patients had a mean age of 67 years (range: 29-89 years), and generally presented symptoms of hypertension and/or hyperlipidemia (Table 1 ). But they rarely had a history of myocardial infarction, prior percutaneous coronary intervention, or a coronary artery bypass graft (CABG). Prospective ECG-gated 1-beat and 2- or 3-beat axial scanning was performed on 241 and 42 patients, respectively. Retrospective ECG-gated helical scan was performed on 26 patients for CABG assessment. The average effective radiation dose was $1.8 \pm 0.4 \mathrm{mSv}, 5.5 \pm$ $1.4 \mathrm{mSv}$, and $18.1 \pm 3.6 \mathrm{mSv}$ for 1-beat-, 2-beat-, or 3beat axial and helical scanning, respectively. Mean contrast material volume used was $43.2 \pm 15.9 \mathrm{~mL}$.

\section{Distribution of appropriateness of cardiac CT}

One hundred and fifty-two of 309 (50\%) patients were classified into appropriate indication, whereas 80 (26\%) and $64(20 \%)$ patients were classified into uncertain and inappropriate indications, respectively (Figure 1). Only 13 scans (4\%) could not be classified. The most common category of cardiac CT indications (29.8\%) was the detection of CAD in symptomatic patients without known heart disease (Table 2). Four other indications accounted for $12 \%-16 \%$ of the patients, including CAD detection 
Table 1 Demographic variables of 309 patients referred for cardiac CT

\begin{tabular}{|c|c|}
\hline \multicolumn{2}{|l|}{ Characteristics } \\
\hline Males & $176(57.0 \%)$ \\
\hline Age & $67.3 \pm 12.3$ years \\
\hline Hypertension, N (\%) & $210(68.0 \%)$ \\
\hline Hyperlipidemia, N (\%) & $148(47.9 \%)$ \\
\hline Diabetes, N (\%) & $74(23.9 \%)$ \\
\hline \multirow[t]{2}{*}{ Smoking history, N (\%) } & Prior: 70 (22.7\%) \\
\hline & Current: 61 (19.7\%) \\
\hline Family history, N (\%) & $160(51.8 \%)$ \\
\hline History of myocardial infarction, N (\%) & $49(15.9 \%)$ \\
\hline Prior $\mathrm{PCl}$ and/or CABG, N (\%) & $38(12.3 \%)$ \\
\hline \multirow[t]{3}{*}{ Tube voltage, N (\%) } & 80 kVp: 24 (7.8\%) \\
\hline & 100 kVp: 185 (59.9\%) \\
\hline & 120 kVp: 100 (32.3\%) \\
\hline \multirow[t]{2}{*}{ Median radiation dose } & $1.9 \mathrm{mSv}$ \\
\hline & (interquartile range, $1.5-2.6 \mathrm{mSv}$ ) \\
\hline
\end{tabular}

Note _ CABG, coronary artery bypass graft; $\mathrm{PCl}$, percutaneous coronary intervention. in other scenarios, and preoperative risk assessment of non-cardiac surgery in the absence of active cardiac conditions. In the latter case, the cardiac CT scan revealed significant coronary arterial stenosis in 20 of 46 patients (43.5\%). A representative case for preoperative risk assessment of non-cardiac surgery is shown in Figure 2a, b and c. The scenarios were rated according to the 2010 AUC system, and the top four indications were rated as appropriate (Table 3). The $5^{\text {th }}$ most frequent indication (5.2\%) was global coronary heart disease risk estimate in asymptomatic/no known CAD patients with intermediate pretest probability, which was rated as inappropriate by AUC 2010.

\section{Relationship of CAD and appropriateness at cardiac CT}

Significant coronary arterial stenosis was detected in 98 of 309 patients. At least one vessel segment with significant stenosis was detected in 42/152 (27.6\%), 34/80 (42.5\%), 20/62 (32.3\%), and $2 / 13$ patients (15.4\%) among the appropriate, uncertain, inappropriate, and not classifiable groups, respectively (Table 4 ). There was a significant difference in the frequency of $>50 \%$ coronary stenosis among the appropriate, uncertain, and inappropriate rating groups $(P=0.03)$, and it was significantly higher in uncertain group than appropriate group. In contrast, the total number of coronary

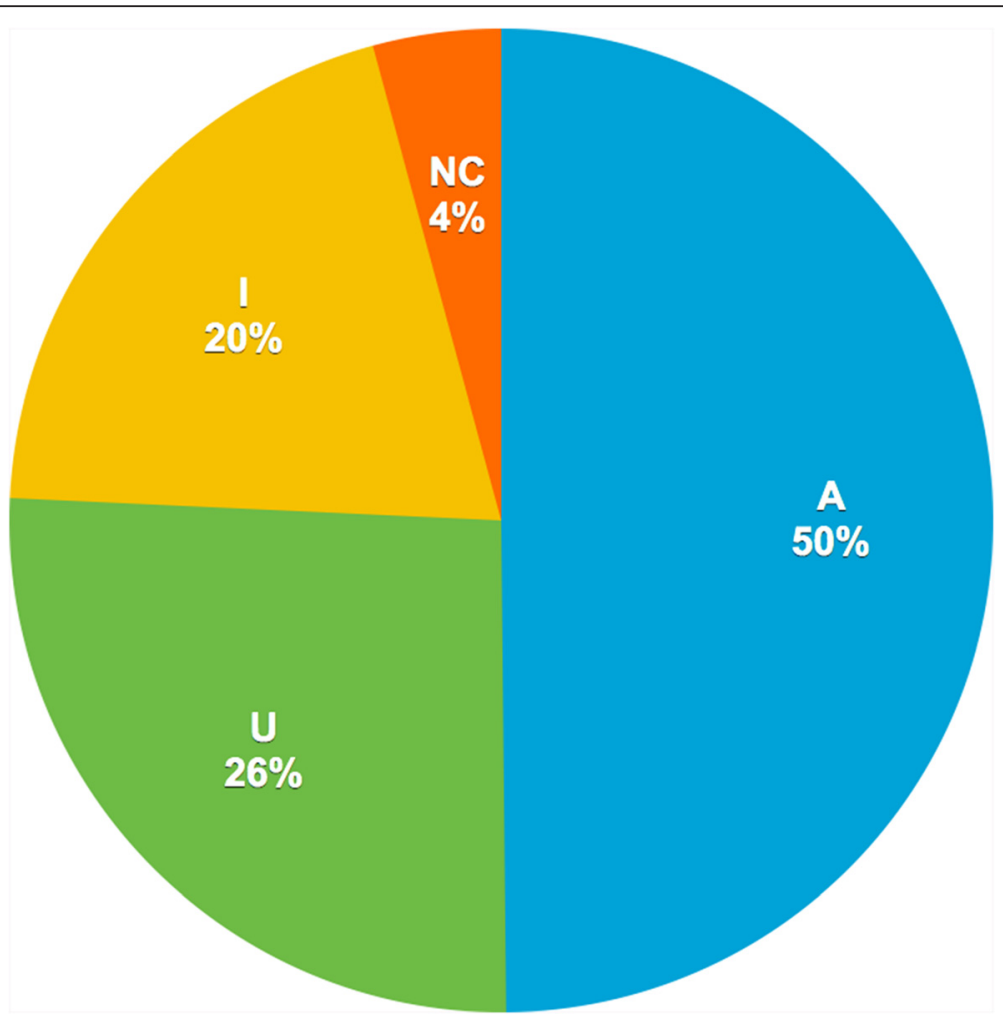

Figure 1 Distribution of patients classified according to the 2010 appropriate use criteria. Note _ A, appropriate; U, uncertain; I, inappropriate; $\mathrm{NC}$, not classifiable. 
Table 2 Category of cardiac CT indications under 2010 appropriate use criteria

\begin{tabular}{ll}
\hline Category & N (\%) \\
\hline $\begin{array}{l}\text { Detection of CAD in symptomatic patients without } \\
\text { known heart disease }\end{array}$ & $92(29.8 \%)$ \\
Detection of CAD/risk assessment & $18(5.8 \%)$ \\
Detection of CAD in other clinical scenarios & $48(15.5 \%)$ \\
Use of CTA in the setting of prior test results & $39(12.6 \%)$ \\
Risk assessment preoperative evaluation of non-cardiac & $46(14.9 \%)$ \\
surgery without acute cardiac condition & $38(12.3 \%)$ \\
Risk assessment post revascularization (PCl or CABG) & $15(4.9 \%)$ \\
Evaluation of cardiac structure and function & $13(4.2 \%)$ \\
Not classifiable & \\
\hline
\end{tabular}

Note _CABG, coronary artery bypass graft; CAD, coronary artery disease; CTA, computed tomography angiography; $\mathrm{PCl}$, percutaneous coronary intervention.

segments with $>50 \%$ stenosis was not significantly different among the groups $(\mathrm{P}=0.13$; Table 5).

\section{Discussion}

This single-academic-center study presents the first attempt to evaluate the applicability of the 2010 AUC to the second-generation 320-row CT in clinical practice. We found that the proportion of uncertain (26\%) rating was higher than that in previous studies (3\% - 16\%) (Cullen et al. 2013; Wasfy et al. 2012; Mazimba et al. 2012). One of the major reasons was the increase in CT examinations for detection of $\mathrm{CAD}$ in asymptomatic

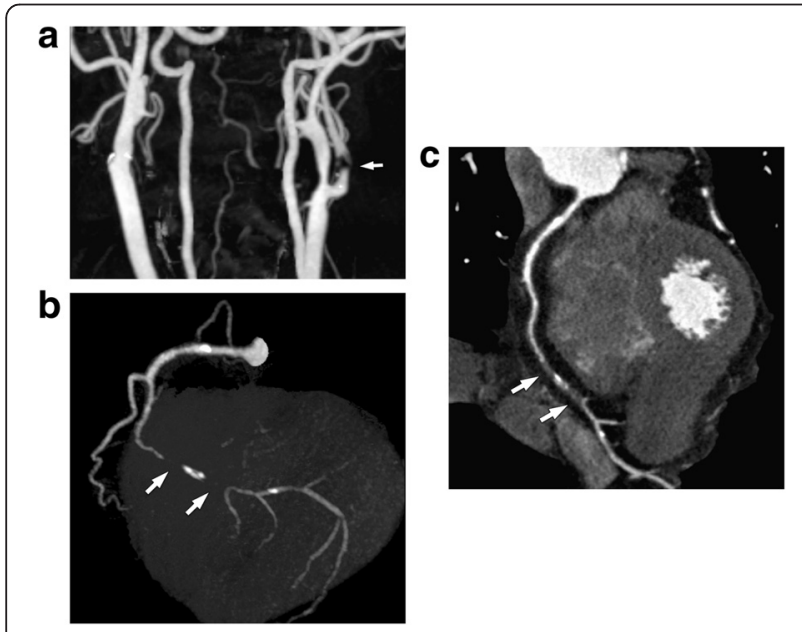

Figure 2 Carotid CT- (a) and coronary CT angiograms (b and c) of a 70-year-old asymptomatic male undergoing carotid endarterectomy. Carotid $C T$ angiogram (a) shows severe stenosis of the left internal carotid artery. For screening of coexisting coronary artery disease, he underwent coronary $\mathrm{CT}$ angiogram prior to carotid endarterectomy. Coronary $\mathrm{CT}$ angiographic images (b): maximum intensity projection image [angiographic view], (c): curved planar reformation image) show a long mixed plaque with severe luminal stenosis in the distal right coronary artery.
Table 3 Most common indications under 2010 appropriate use criteria

2010 AUC: most frequent indications

Non-acute symptoms possibly representing an ischemic equivalent- interpretable ECG AND able

to exercise-intermediate pretest probability (A)

Reduced left ventricular ejection fraction-low or intermediate pretest probability $(A)$

CTA after prior stress imaging procedure deemed equivocal (A)

Non-acute symptoms possibly representing an ischemic equivalent-uninterpretable ECG OR unable to exercise (A)

Global CHD risk estimate-asymptomatic/no known CAD-low or intermediate pretest probability (I)

Note _ A, appropriate; AUC, appropriate use criteria; CAD, coronary artery disease; CHD, coronary heart disease; CTA, computed tomography angiography; ECG, electrocardiogram; I, inappropriate; $\mathrm{PCl}$, prior percutaneous coronary intervention; $U$, uncertain.

patients. The popularity of cardiac CT to screen patients with carotid artery stenosis and peripheral artery disease is increasing because these patients usually have multiple risk factors, including atherosclerosis. Among patients undergoing carotid endarterectomy or carotid artery stenting, $30 \%-50 \%$ of those with carotid artery stenosis had significant CAD (Shimada et al. 2005; Enomoto et al. 2013), and the severity of carotid artery stenosis and the extent of CAD were significantly correlated (Steinvil et al. 2011). In our survey, $43.5 \%$ of asymptomatic patients undergoing cardiac CT for preoperative evaluation of non-cardiac surgery had significant coronary artery stenosis. This might explain why the presence of significant $C A D$ was higher in the uncertain rating group than in the appropriate rating group. We believe that non-invasive assessment of coexisting advanced atherosclerosis and CAD using current CT technology should be clinically important and appropriate for reducing perioperative myocardial infarction and stroke. The American College of Cardiology Foundation recognizes the importance of revising the criteria in timely manner in order to provide the cardiovascular community with the accurate indications (Taylor et al. 2010). Many uncertain-rating indications under the 2006 AUC changed to appropriate-rating under the 2010 AUC (Rich et al. 2012). Some of current uncertain-rating scenarios may potentially change to appropriate rating in the near future according to the rapid advances in CT technology.

Compared with invasive coronary angiography, cardiac CT using 64-row CT shows high diagnostic accuracy for detecting significant CAD (Sun et al. 2008). However, the widespread use of cardiac $\mathrm{CT}$ raises concerns regarding radiation exposure. With retrospective ECG-gated 64-row $\mathrm{CT}$, the mean effective radiation dose is approximately 15 $\mathrm{mSv}$ (Alkadhi and Leschka 2011). While the radiation dose can be reduced by more than $50 \%$ with the step-and-shoot 
Table 4 Significant CAD among 309 patients referred for cardiac CT

\begin{tabular}{llll}
\hline 2010 AUC & No lesion [N (\%)] & At least one vessel with $\mathbf{~ 5 0 \% ~ s t e n o s i s ~ [ N ~ ( \% ) ] ~}$ & Unable to evaluate [N (\%)] \\
\hline Appropriate $(\mathrm{N}=154)$ & $95(30.7 \%)$ & $42(13.6 \%)$ & $17(5.5 \%)$ \\
Uncertain $(\mathrm{N}=80)$ & $34(11.0 \%)$ & $34(11.0 \%)$ & $10(3.3 \%)$ \\
Inappropriate $(\mathrm{N}=62)$ & $33(10.7 \%)$ & $20(6.4 \%)$ & $11(3.6 \%)$ \\
Not classifiable $(\mathrm{N}=13)$ & $10(3.3 \%)$ & $2(0.6 \%)$ & $1(0.3 \%)$ \\
\hline
\end{tabular}

Note _ AUC, appropriate use criteria; CAD, coronary artery disease.

mode, this technique can only be used in patients strictly defined by their heart rate, heart rate variations, and body habitus. In our study, 241 of 309 (78\%) patients underwent 1-beat prospective axial scan with iterative reconstruction technique, and their mean effective dose was only 1.8 $\mathrm{mSv}$. In addition, we used 100 - and $80-\mathrm{kVp}$ scanning in $60 \%$ and $8 \%$ of patients, respectively. Low-voltage $(80-100$ $\mathrm{kVp}$ ) techniques drastically reduce the radiation dose and effectively increase vascular enhancement (Hoffmann et al. 2012). Cardiac CT imaging plus iterative reconstruction reduces the radiation dose by $55 \%$ while yielding a contrast-to-noise ratio equal to $120-\mathrm{kVp} \mathrm{CT}$ imaging with the filtered back projection reconstruction (Hoffmann et al. 2012; Hou et al. 2012). In our study population, the mean contrast material volume was around $40 \mathrm{~mL}$ by using low-voltage technique and iterative reconstruction. A previous study (Rich et al. 2012) reported that lowvoltage $(100 \mathrm{kVp})$ cardiac CT was performed in 313 of 1293 (26\%) patients between 2010 and 2011. In the present study, we used low-voltage cardiac scanning in $57 \%$ of patients. We consider that the application of iterative reconstruction for the smaller body habitus of Asians might result in a higher percentage of low-voltage CT. A previous study (Layritz et al. 2013) suggested that an automated attenuation-based selection of tube voltage and tube current can be used in clinical cardiac CT. We postulate that the appropriate selection of scan parameters with iterative reconstruction technique can increase the percentage of low-voltage CT, and reduce radiation exposure even in heavier patients in Western countries. Under these conditions, less-invasive cardiac CT would become more readily available, safe, and beneficial for patients with possible CAD, resulting in increase of clinical indications and appropriateness.
Our study has some limitations. First, we counted $>50 \%$ stenosis as significant $\mathrm{CAD}$ on $\mathrm{CT}$, but did not compare the CT finding with myocardial perfusion imaging. Stenosis of $>50 \%$ does not directly mean myocardial ischemia. Also, we did not assess the net reclassification index of each appropriateness category. The proportion of obstructive CAD changes by the pre-test risk. Therefore, the assessment of the ability of cardiac CT to reclassify the risk of obstructive CAD should be important. Further studies should be performed to validate the relationship between uncertain category of the 2010 AUC and obstructive CAD. Second, our single-center study population was relatively small. Third, we did not compare the AUC rating between 64-row- and the updated 320-row machines. We posit that the differences between the previous study and our results might reflect our less-invasive cardiac CT influenced the clinical practice in the management of CAD patients. Fourth, we did not assess the interobserver agreement of the 2010 AUC ratings. Nonetheless, the ratings were based on consensus agreement between two experienced radiologists, and a very good interobserver agreement has been reported for this type of evaluation. Fifth, we did not compare image quality between the second- and first-generation 320-row CT. We believe that the updated 320-row CT provides better image quality due to a reduction in motion artifacts. Lastly, our interpretation of the cardiac CT images was not blinded to patient information. However, appropriateness ratings were determined before image interpretation and reporting.

\section{Conclusion}

Cardiac CT with the second-generation 320-row CT may influence patient selection, leading to fewer indications

Table 5 Number of coronary segments with significant stenosis among patients with significant coronary arterial stenosis

\begin{tabular}{llll}
\hline $\mathbf{2 0 1 0}$ AUC & \multicolumn{2}{l}{ Number of coronary segments with $\mathbf{> 5 0 \%}$ stenosis } \\
\cline { 2 - 4 } & $\mathbf{1}$ segment & $\mathbf{2 ~ s e g m e n t s}$ & $\mathbf{3}$ or more segments \\
\hline Appropriate $(\mathrm{N}=42)$ & $22(22.5 \%)$ & $7(7.1 \%)$ & $13(13.3 \%)$ \\
Uncertain $(\mathrm{N}=34)$ & $16(16.3 \%)$ & $5(5.1 \%)$ & $13(13.3 \%)$ \\
Inappropriate $(\mathrm{N}=20)$ & $14(14.3 \%)$ & $2(2.0 \%)$ & $4(4.1 \%)$ \\
Not classifiable $(\mathrm{N}=2)$ & 0 & 0 & $2(2.0 \%)$ \\
\hline
\end{tabular}

Note _ AUC, appropriate use criteria. 
that could not be classified, but a substantial increase in the number of uncertain scans. According to the 2010 AUC, uncertain rating is associated with a high prevalence of CAD. Our study may suggest that clinical indications of cardiac CT may become wider with the advances of the CT scanner and technology, resulting in early detection of significant CAD.

\section{Competing interests}

The authors declare that they have no competing interests.

\section{Authors' contributions}

DU designed and carried out the clinical study, interpreted the data, drafted and revised the manuscript for intellectual content. SO, HY, MY, KT, MY and MK supported the data collection, and drafted the manuscript. YF performed statistical analysis. $\mathrm{HO}$ and $\mathrm{YY}$ drafted and revised the manuscript for intellectual content. All authors read and approved the final manuscript.

\section{Author details}

${ }^{1}$ Diagnostic Radiology, Faculty of Life Sciences, Kumamoto University, 1-1-1, Honjo, 860-8556 Kumamoto-shi, Kumamoto, Chuo-ku, Japan. ${ }^{2}$ Cardiovascular Medicine, Faculty of Life Sciences, Kumamoto University, Kumamoto, Japan. ${ }^{3}$ Medical Physics, Faculty of Life Sciences, Kumamoto University, Kumamoto, Japan.

Received: 25 October 2014 Accepted: 30 January 2015

Published online: 05 March 2015

\section{References}

Alkadhi H, Leschka S (2011) Radiation dose of cardiac computed tomography what has been achieved and what needs to be done. Eur Radiol 21(3):505-509, doi:10.1007/s00330-010-1984-3

Chen MY, Shanbhag SM, Arai AE (2013) Submillisievert median radiation dose for coronary angiography with a second-generation 320-detector row CT scanner in 107 consecutive patients. Radiology 267(1):76-85, doi:10.1148/radiol.13122621

Cullen EL, Aggarwal SR, Goss BC, Hodge DO, Gibbons RJ, Araoz PA (2013) Comparison of the applicability of the 2006 and the 2010 cardiac CT angiography appropriate use criteria. J Am Coll Radiol JACR 10(4):258-267, doi:10.1016/j.jacr.2012.07.014

Enomoto Y, Yoshimura S, Yamada K, Kawasaki M, Nishigaki K, Minatoguchi S, Iwama T (2013) Silent coronary artery disease in Japanese patients undergoing carotid artery stenting. J Stroke Cerebrovasc Dis 22(7):1163-1168, doi:10.1016/j.jstrokecerebrovasdis.2012.12.013

Hausleiter J, Meyer T, Hermann F, Hadamitzky M, Krebs M, Gerber TC, McCollough C, Martinoff S, Kastrati A, Schomig A, Achenbach S (2009) Estimated radiation dose associated with cardiac CT angiography. JAMA 301 (5):500-507, doi:10.1001/jama.2009.54

Hendel RC, Patel MR, Kramer CM, Poon M, Hendel RC, Carr JC, Gerstad NA, Gillam LD, Hodgson JM, Kim RJ, Kramer CM, Lesser JR, Martin ET, Messer JV, Redberg RF, Rubin GD, Rumsfeld JS, Taylor AJ, Weigold WG, Woodard PK, Brindis RG, Hendel RC, Douglas PS, Peterson ED, Wolk MJ, Allen JM, Patel MR, American College of Cardiology Foundation Quality Strategic Directions Committee Appropriateness Criteria Working G, American College of R, Society of Cardiovascular Computed T, Society for Cardiovascular Magnetic R, American Society of Nuclear C, North American Society for Cardiac I, Society for Cardiovascular A, Interventions, Society of Interventional R (2006) ACCF/ ACR/SCCT/SCMR/ASNC/NASCI/SCAI/SIR 2006 appropriateness criteria for cardiac computed tomography and cardiac magnetic resonance imaging: a report of the American College of Cardiology Foundation Quality Strategic Directions Committee Appropriateness Criteria Working Group, American College of Radiology, Society of Cardiovascular Computed Tomography, Society for Cardiovascular Magnetic Resonance, American Society of Nuclear Cardiology, North American Society for Cardiac Imaging, Society for Cardiovascular Angiography and Interventions, and Society of Interventional Radiology. J Am Coll Cardiol 48(7):1475-1497, doi:10.1016/j.jacc.2006.07.003 Hoffmann U, Venkatesh V, White RD, Woodard PK, Carr JJ, Dorbala S, Earls JP, Jacobs JE, Mammen L, Martin ET 3rd, Ryan T, White CS (2012) ACR appropriateness
criteria((R)) acute nonspecific chest pain-low probability of coronary artery disease. J Am Coll Radiol JACR 9(10):745-750, doi:10.1016/j.jacr.2012.06.032 Hou Y, Liu X, Xv S, Guo W, Guo Q (2012) Comparisons of image quality and radiation dose between iterative reconstruction and filtered back projection reconstruction algorithms in 256-MDCT coronary angiography. AJR Am J Roentgenol 199(3):588-594, doi:10.2214/AJR.11.7557

Khan A, Khosa F, Nasir K, Yassin A, Clouse ME (2011) Comparison of radiation dose and image quality: 320-MDCT versus 64-MDCT coronary angiography. AJR Am J Roentgenol 197(1):163-168, doi:10.2214/AJR.10.5250

Layritz C, Muschiol G, Flohr T, Bietau C, Marwan M, Schuhbaeck A, Schmid J, Ropers D, Achenbach S, Pflederer T (2013) Automated attenuation-based selection of tube voltage and tube current for coronary $\subset$ angiography: reduction of radiation exposure versus a BMl-based strategy with an expert investigator. J Cardiovasc Comput Tomogr 7(5):303-310, doi:10.1016/j.jcct.2013.08.010

Leipsic J, Heilbron BG, Hague C (2012) Iterative reconstruction for coronary CT angiography: finding its way. Int J Cardiovasc Imaging 28(3):613-620, doi:10.1007/s10554-011-9832-3

Mazimba S, Grant N, Parikh A, Patel T, Dahale B, Franco Z, Dittoe N, Shah T, Hahn HS (2012) Comparison of the 2006 and 2010 cardiac CT appropriateness criteria in a real-world setting. J Am Coll Radiol JACR 9(9):630-634, doi:10.1016/j.jacr.2012.04.016

Nakaura T, Kidoh M, Sakaino N, Utsunomiya D, Oda S, Kawahara T, Harada K, Yamashita Y (2013) Low contrast- and low radiation dose protocol for cardiac $C T$ of thin adults at 256-row CT: usefulness of low tube voltage scans and the hybrid iterative reconstruction algorithm. Int J Cardiovasc Imaging 29 (4):913-923, doi:10.1007/s10554-012-0153-y

Oda S, Utsunomiya D, Funama Y, Katahira K, Honda K, Tokuyasu S, Vembar M, Yuki H, Noda K, Oshima S, Yamashita Y (2014) A knowledge-based iterative model reconstruction algorithm: can super-low-dose cardiac ct be applicable in clinical settings? Acad Radiol 21(1):104-110, doi:10.1016/j.acra.2013.10.002

Rich ME, Utsunomiya D, Simprini LA, Weigold WG, Weissman G, Taylor AJ (2012) Prospective evaluation of the updated 2010 ACCF Cardiac CT Appropriate Use Criteria. J Cardiovasc Comput Tomogr 6(2):108-112, doi:10.1016/j.jcct.2012.02.003

Shimada T, Toyoda K, Inoue T, Kamouchi M, Matsumoto T, Hiyamuta K, Imaizumi T, Okada Y (2005) Prediction of coronary artery disease in patients undergoing carotid endarterectomy. J Neurosurg 103(4):593-596, doi:10.3171/jns.2005.103.4.0593

Steinvil A, Sadeh B, Arbel Y, Justo D, Belei A, Borenstein N, Banai S, Halkin A (2011) Prevalence and predictors of concomitant carotid and coronary artery atherosclerotic disease. J Am Coll Cardiol 57(7):779-783, doi:10.1016/j.jacc.2010.09.047

Sun Z, Lin C, Davidson R, Dong C, Liao Y (2008) Diagnostic value of 64-slice CT angiography in coronary artery disease: a systematic review. Eur J Radiol 67(1):78-84, doi:10.1016/j.ejrad.2007.07.014

Tatsugami F, Matsuki M, Nakai G, Inada Y, Kanazawa S, Takeda Y, Morita H, Takada H, Yoshikawa S, Fukumura K, Narumi Y (2012) The effect of adaptive iterative dose reduction on image quality in 320-detector row $C T$ coronary angiography. Br J Radiol 85(1016):e378-e382, doi:10.1259/bjr/10084599

Taylor AJ, Cerqueira M, Hodgson JM, Mark D, Min J, O'Gara P, Rubin GD, Kramer CM, Berman D, Brown A, Chaudhry FA, Cury RC, Desai MY, Einstein AJ, Gomes AS, Harrington R, Hoffmann U, Khare R, Lesser J, McGann C, Rosenberg A, Schwartz R, Shelton M, Smetana GW, Smith SC Jr (2010) ACCF/ SCCT/ACR/AHA/ASE/ASNC/NASCI/SCAI/SCMR 2010 appropriate use criteria for cardiac computed tomography. A report of the American College of Cardiology Foundation Appropriate Use Criteria Task Force, the Society of Cardiovascular Computed Tomography, the American College of Radiology, the American Heart Association, the American Society of Echocardiography, the American Society of Nuclear Cardiology, the North American Society for Cardiovascular Imaging, the Society for Cardiovascular Angiography and Interventions, and the Society for Cardiovascular Magnetic Resonance. J Am Coll Cardiol 56(22):1864-1894, doi:S0735-1097(10)02529-5

Wasfy MM, Brady TJ, Abbara S, Nasir K, Hoffmann U, Cury RC, Di Carli MF, Blankstein R (2012) Comparison of cardiac computed tomography examination appropriateness under the 2010 revised versus the 2006 original appropriate use criteria. J Cardiovasc Comput Tomogr 6(2):99-107, doi:10.1016/j.jcct.2011.12.005 